\title{
Effect of the human SOD1-G93A gene on the Nrf2/ARE signaling pathway in NSC-34 cells
}

\author{
FUMIN WANG ${ }^{1,2}$, YUCHENG LU ${ }^{3}$, FAYING QI $^{2}$, QUANPING SU $^{3}$, LONG WANG $^{3}$, \\ CUIPING YOU ${ }^{3}$, FENGYUAN $\mathrm{CHE}^{2}$ and JIXU YU ${ }^{2}$ \\ ${ }^{1}$ Department of Clinical Neurology, School of Neurology, Weifang Medical University, Weifang, Shandong 261053; \\ ${ }^{2}$ Department of Neurology; ${ }^{3}$ Central Laboratory, Linyi People's Hospital, Linyi, Shandong 276003, P.R. China
}

Received October 12, 2013; Accepted March 4, 2014

DOI: $10.3892 / \mathrm{mmr} .2014 .2087$

\begin{abstract}
Dominant mutations in superoxide dismutase 1 (SOD1) are a frequent cause of the lethal neurodegenerative disease amyotrophic lateral sclerosis (ALS). The nuclear factor erythroid 2-related factor 2 (Nrf2)/antioxidant response element (ARE) signaling pathway is the major cellular defense mechanism against oxidative stress, however, its role in ALS remains to be fully elucidated. Therefore, the present study aimed to investigate whether the human SOD1-G93A gene affected the Nrf2/ARE signaling pathway in an ALS cell model. The soma became round and the number of neurites decreased in the NSC-34 cells transfected with the hSOD1-G93A gene, and the neurites were shorter and oxidative stress was increased compared with the normal NSC-34 cells. Furthermore, the mRNA and protein expression of Nrf2, heme oxygenase-1 and $\mathrm{NAD}(\mathrm{P}) \mathrm{H}$ : quinone oxidoreductase 1 was significantly decreased in the NSC-34 cells transfected with the human SOD1-G93A gene. The present study indicated that human SOD1-G93A damaged the Nrf2/ARE signaling pathway in the ALS cell model and reduced the ability of cells to protect against oxidative injury.
\end{abstract}

\section{Introduction}

Amyotrophic lateral sclerosis (ALS) is a lethal neurodegenerative disease characterized by the progressive loss of motor neurons in the motor cortex, brainstem and spinal cord (1). The clinical manifestations include the progressive development of major muscle weakness, atrophy, tremors, tendon reflexes hyperfunction and pathologically positive features.

Correspondence to: Mr. Jixu $\mathrm{Yu}$ or Mr. Fengyuan Che, Department of Neurology, Linyi People's Hospital, 27 Jiefang Road, Linyi, Shandong 276003, P.R. China

E-mail: yujixu@yahoo.com

E-mail: che1971@126.com

Key words: amyotrophic lateral sclerosis, neurodegenerative disease, NSC-34 cells, oxidative stress, Nrf2/ARE pathway, phase II enzymes
Approximately $90-95 \%$ of all ALS cases are sporadic and $5-10 \%$ of cases are familial (fALS). At present, the pathogenesis of ALS remains to be fully elucidated and there are no effective treatment methods. The majority of ALS patients succumb to respiratory failure within 3-5 years of clinical onset (2).

Present evidence suggests $(3,4)$ that the progressive loss of motor neurons in ALS cases results from a series of complex interaction mechanisms, including oxidative stress, excitotoxicity, altered axonal transport, mitochondrial dysfunction, an abnormal cytoskeleton, protein aggregation and genetic factors. In these mechanisms, oxidative stress is considered to be important in the pathogenesis of selective motor neuron degeneration and corticospinal tract degeneration (5), and it is also a major contributory factor leading to chronic motor neuron degeneration and death (6). Several previous studies have demonstrated that oxidative stress is present in cerebrospinal fluid and spinal cord slices of ALS patients, suggesting that oxidative stress may be a crucial link in the pathogenesis of ALS (5). In mutant superoxide dismutase 1 (SOD1) ALS mouse models, the level of oxidative damage to biological macromolecules (including proteins, lipids and nucleic acid) was elevated in the brain and spinal cord. Numerous previous studies have revealed that $\sim 20 \%$ of cases of fALS are caused by mutations in the antioxidant enzyme $\mathrm{Cu} / \mathrm{Zn}$ SOD1 (7). Rodents, which overexpress mutant forms of hSOD1, usually develop an ALS-like phenotype $(8,9)$. At present, the toxic pathogenic mechanisms for human SOD1 mutant transgenic animals remain unknown. A previous study found that nuclear factor erythroid 2-related factor 2 (Nrf2) was able to interact with the antioxidant response element (ARE) to regulate the expression of phase II antioxidant enzymes including $\mathrm{NAD}(\mathrm{P}) \mathrm{H}$ : quinone oxidoreductase 1 (NQO1) and heme oxygenase-1 (HO-1). The Nrf2/ARE signaling pathway is one of the most important endogenous antioxidant stress pathways (10). Previous studies have demonstrated that the activation of the Nrf2/ARE signaling pathway was able to decrease different types of cellular damage in different tissues and organs (11-13). At present, the role of the Nrf2/ARE signaling pathway in the onset of ALS motor neuron degeneration remains to be fully elucidated. In the present study, the effect of the mutant human SOD1-G93A gene on the Nrf2/ARE endogenous antioxidant pathway was observed 
using NSC-34 cells transfected with human SOD1-G93A, which was already established in our laboratory. The present study aimed to provide a further theoretical basis for the pathogenesis and treatment of ALS.

\section{Materials and methods}

Chemicals. An antibody recognizing neurofilament (SMI-32) was purchased from Covance Inc. (Princeton, NJ, USA). Other antibodies, including anti- $\beta$-actin, anti-Nrf2, anti-NQO1 and anti-HO-1 were purchased from Santa Cruz Biotechnology, Inc. (Santa Cruz, CA, USA). A First Strand cDNA Synthesis kit was purchased from Thermo Fisher Scientific (Waltham, MA, USA). A malondialdehyde (MDA) assay kit was purchased from Nanjing Jiancheng Bioengineering Institute (Nanjing, Jiangsu, China). A total protein extraction kit was purchased from Nanjing KeyGen Biotech., Co., Ltd (Nanjing, Jiangsu, China).

Cell lines and cell cultures. NSC-34 is a hybrid cell line, produced by fusion of motor neuron enriched, embryonic mouse spinal cord cells with mouse neuroblastoma, which possesses several of the unique morphological and physiological characteristics of motor neurons. NSC-34 cells stably transfected with the empty pcDNA3.1(-) plasmid and the pcDNA3.1(-) plasmid carrying hSOD1-WT and hSOD1-G93A were successfully established. The cell lines were removed from liquid nitrogen and thawed quickly in a $37^{\circ} \mathrm{C}$ water bath. Then, the cells were diluted 5 -fold with complete medium (containing 90\% Dulbecco's modified Eagle's medium, 10\% fetal bovine serum, $100 \mathrm{IU} / \mathrm{ml}$ penicillin and $0.1 \mathrm{mg} / \mathrm{ml}$ streptomycin) and seeded to $25 \mathrm{~cm}^{2}$ glass culture bottles. The cultures were maintained at $37^{\circ} \mathrm{C}$ in a $5 \% \mathrm{CO}_{2}$ humidified atmosphere. Following attachment of the cells to the culture bottles ( 4-6 h), the medium was refreshed to remove the dimethyl sulfoxide. The medium was generally changed every 2-3 days, depending on the rate of growth.

Immunocytochemical analysis. An immunocytochemical staining technique was used to observe the morphology of stably transfected cells. The stably transfected cells were digested and collected by centrifugation and then resuspended with culture medium. Then, the cells were seeded in a 6 -well culture plate at a density of $2 \times 10^{4} / \mathrm{ml}$. Following $24 \mathrm{~h}$, the cells were rinsed with $0.01 \mathrm{M}$ phosphate-buffered saline (PBS) and fixed with $4 \%$ paraformaldehyde for $40 \mathrm{~min}$. To inhibit non-specific binding, the cells were incubated with goat serum incubated for $15 \mathrm{~min}$. The cultures were incubated with an anti-neurofilament antibody (SMI-32) overnight at $4^{\circ} \mathrm{C}$. The cultures were washed three times with PBS and incubated with a biotinylated secondary antibody for $15 \mathrm{~min}$ in $37^{\circ} \mathrm{C}$ and then visualized with $3,3^{\prime}$-diaminobenzidine tetrahydrochloride.

Measurement of MDA. MDA is one of the most important degradation products of lipid peroxidation. It is able to react with thiobarbituric acid to generate mauve material. The enzyme activity was determined by monitoring the change in absorbance at $532 \mathrm{~nm}$.
Reverse transcription polymerase chain reaction. Total RNA was isolated using TRIzol reagent. The concentrations and qualities of RNA were determined by measuring absorbance at 260 and $280 \mathrm{~nm}$. The synthesis of the first chain cDNA was performed according to the manufacturer's instructions of the First Strand cDNA Synthesis kit (Thermo Fisher Scientific). A $25 \mu 1$ system of PCR amplification was used and consisted of $12.5 \mu 1$ of DreamTaq Green PCR Master mix (2X), $10.5 \mu 1$ of sterile ultrapure water, $0.5 \mu 1$ of cDNA samples and $0.5 \mu 1$ of forward and reverse primers. The specific primers used were as follows: $\beta$-actin, forward 5'-GGGACCTGACTGACTACCTCA-3' and reverse 5'-GACTCGTCATACTCCTGCTTG-3'; Nrf2, forward 5'-ATCGACAGTGCTCCTATGCGTGAA-3' and reverse 5'-ATCGTCTGGGCGGCGACTTTAT-3'; HO-1, forward 5'-ATCGTGGTGATGGCTTCCTTGT-3' and reverse 5'-ATCGACCTCGTGGAGACGCTTT-3'; NQO1, forward 5'-ATCGGAGAAGAGCCCTGATTGT-3' and reverse 5'-ATCGAAAGGACCGTTGTCGTAC-3'. For PCR, the amplification conditions consisted of an initial activation step at $95^{\circ} \mathrm{C}$ for $4 \mathrm{~min}$ and 30 cycles at $95^{\circ} \mathrm{C}$ for $15 \mathrm{sec}, 60^{\circ} \mathrm{C}$ for $15 \mathrm{sec}, 72^{\circ} \mathrm{C}$ for $30 \mathrm{sec}$ and finally $72^{\circ} \mathrm{C}$ for $7 \mathrm{~min}$. In order to detect the reverse transcription PCR amplification products, $1.5 \%$ agarose gel electrophoresis was used.

Western blot analysis. The proteins were extracted using a total protein extraction kit. The extraction of protein was quantified using the bradford method. A total of $60 \mathrm{mg}$ of extracted protein was resolved by $10 \%$ sodium dodecyl sulfate-polyacrylamide gel electrophoresis and the resolved proteins in the gel were transferred onto polyvinylidene difluoride membranes. The membranes were incubated overnight at $4^{\circ} \mathrm{C}$ with the following specific primary antibodies: rabbit polyclonal anti-Nrf2 (1:200), rabbit polyclonal anti-HO-1 (1:200), goat polyclonal anti-NQO1 (1:200) and mouse monoclonal anti- $\beta$-actin (1:500). Then, the membranes were incubated with corresponding secondary antibody (the dilution of $\beta$-actin secondary antibody was $1: 10,000$ and the others were $1: 3,000)$ for $1 \mathrm{~h}$ at room temperature and immunodetection was performed with an enhanced chemiluminescent substrate. The data calculated were obtained by the rate of the density of target protein banding to the density of corresponding $\beta$-actin banding.

Statistical analysis. The results are expressed as the mean \pm standard deviation. Statistical analyses were performed using two-way analysis of variance. $\mathrm{P}<0.05$ was considered to indicate a statistically significant difference.

\section{Results}

Effect of the human SOD1-G93A gene on NSC-34 cellular morphology. In order to observe the morphological alterations of cells stably transfected with the pcDNA3.1(-) plasmid, the hSOD1-pcDNA3.1(-) plasmid and the hSOD1-G93A-pcDNA3.1(-) plasmid, the stably transfected cells were dyed and examined using the immunocytochemical method. Observations under the microscope revealed that the soma exhibited a rounded morphology and the number of neurites decreased in the NSC-34 cells transfected with 
the hSOD1-G93A gene. In addition, the neurites were shorter compared with the normal NSC-34 cells. However, no significant changes in the cells transfected with the pcDNA3.1(-) and the hSOD1-pcDNA3.1(-) plasmid were identified (Fig. 1).

Detection of the lipid peroxidation level. MDA is able to react with thiobarbituric acid to generate mauve material. The content of MDA directly reflects the level of lipid peroxidation in cells. The present study found that the content of MDA was $2.74 \pm 0.14 \mathrm{nmol} / \mathrm{mg}$ protein in NSC-34 cells which were stably transfected with the hSOD1-G93A gene. The content of MDA in the normal, empty and hSOD1-WT cells was $1.15 \pm 0.53$, $1.65 \pm 0.21$ and $1.81 \pm 0.18 \mathrm{nmol} / \mathrm{mg}$ prot, respectively. Statistical analysis demonstrated that the content of MDA in NSC-34 cells transfected with the hSOD1-G93A gene was significantly increased compared with the other three cell lines, while no significant difference in MDA content in the other three cell lines was observed (Fig. 2).

Effect of the hSOD1-G93A gene on the mRNA expression of Nrf2 and phase II antioxidant enzymes in NSC-34 cells. Previous studies and the present study revealed that the level of oxidative stress in NSC-34 cells transfected with the hSOD1-G93A gene significantly increased. In order to examine whether increasing oxidative stress was associated with the effect of hSOD1-G93A on the Nrf2/ARE signaling pathway, the difference in the mRNA expression of Nrf2 and phase II enzymes was detected in four types of cell lines. The mRNA expression of Nrf2 in NSC-34 cells transfected with the hSOD1-G93A gene markedly decreased compared with the other three cell lines (Fig. 3A and B). Further studies also revealed that the mRNA expression of the downstream factors HO-1 and NQO1 decreased (Fig. 3A, C and D). By contrast, no significant differences in the cells transfected with pcDNA3.1 (-) and hSOD1-WT compared with the normal NSC-34 cells were identified. These results demonstrated that the Nrf2/ARE signaling pathway was possibly affected by hSOD1-G93A.

Effect of the hSOD1-G93A gene on the protein expression of Nrf2 and phase II antioxidant enzymes in NSC-34 cells. In order to further observe the effect of hSOD1-G93A on the Nrf2/ARE signaling pathway in NSC-34 cells, the differences in the protein expression of $\mathrm{Nrf} 2$ and phase II enzymes in these four types of cell lines were detected. The western blots demonstrated that the protein expression of Nrf2 in NSC-34 cells transfected with the hSOD1-G93A gene markedly decreased compared with the other three cell lines (Fig. 4A and B; $\mathrm{P}<0.01$ ). Furthermore, the protein expression of the downstream factors HO-1 and NQO1 decreased as well (Fig. 4C-F; P<0.01). However, no significant difference in the protein expression of Nrf2, $\mathrm{HO}-1$ and NQO1 in the other three cell lines was identified. This phenomenon was consistent with the results from the genetic test.

\section{Discussion}

In order to investigate the toxic effect of hSOD1-G93A on motor neurons, the NSC-34 cell lines were selected and the effect of the hSOD1-G93A gene on the Nrf2/ARE endogenous antioxidant pathway was observed. NSC-34 cells transfected with hSOD1-G93A were already established and commonly used in our laboratory. The present study found that the soma exhibited a rounded morphology and the number of neurites decreased in the NSC-34 cells transfected with the hSOD1-G93A gene. In addition, the neurites were shorter compared with the normal NSC-34 cells. Furthermore, the hSOD1-G93A gene reduced the ability of cells to protect against oxidative damage and increased the levels of lipid peroxidation in cells. Oxidative damage has been substantiated by the biochemical and histopathological study in postmortem tissue of ALS patients $(14,15)$. These studies revealed that the levels of lipid peroxidation, nucleic acid oxidation and protein nitration in ALS patients significantly increased. In order to observe the degree of oxidative damage, the intracellular MDA levels in normal, empty, hSOD1-WT and hSOD1-G93A cell lines were detected. The results from the present study demonstrated that the intracellular MDA levels in hSOD1-G93A cell lines significantly increased compared with the other three cell lines (Fig. 2). This result indicated that the activity of antioxidants and detoxification of the cell lines transfected with the hSOD1-G93A gene decreased.

Furthermore, the present study found that the mRNA expression of Nrf2 in NSC-34 cells, which was stably transfected with the hSOD1-G93A gene, markedly decreased compared with the other three cell lines (Fig. 3A and B). In addition, the mRNA expression of the downstream factors HO-1 and NQO1 also decreased (Fig. 3A, C and D). To further investigate the effect of hSOD1-G93A on the Nrf2/ARE signaling pathway in cells, the western blots of total cellular protein indicated that the protein expression of Nrf2, HO-1 and NQO1 significantly decreased in the mutated hSOD1 NSC-34 cell lines (Fig. 4). This is consistent with the result obtained by Sarlette et al (16). The authors reported that the protein expression of Nrf2 was reduced in neurons from the primary motor cortex and spinal cord from ALS postmortem tissue samples. In addition, the level of mRNA encoding Nrf2 was decreased in embryonic motor neurons isolated from hSOD1-G93A rats (17). Petri et al (6) hypothesized that the decrease in the protein expression of Nrf2 in motor neurons was possibly associated with the decline in the antioxidant defense mechanism of the body. Under basal conditions, the Nrf2 was usually bound to the endogenous inhibitor Kelch-like ECH associated protein 1 (Keap1) in the cell plasma and was in the inactive state. However, when the cells were exposed to oxidative stress, Nrf2 was able to dissociate from the Keap1 protein and translocate to the nucleus, and then bind to ARE and activate the expression of target genes, including HO-1 and NQO1 $(18,19)$. Since the cells demonstrated a high vulnerability to oxidative damage in the HO-1-/- mouse model, HO-1 was considered to be important in the process of endogenous defense against oxidative stress (20). Certain studies suggested that NQO1 was directly able to protect tissues against oxidative stress (21). In cells, NQO1 was a type of electronic reductase regulating substances in the redox state and was able to catalyze the reduction reaction of Quinones and its derivatives. The characteristics of the reaction process did not generate these products, including free radical oxidation and half quinone, 

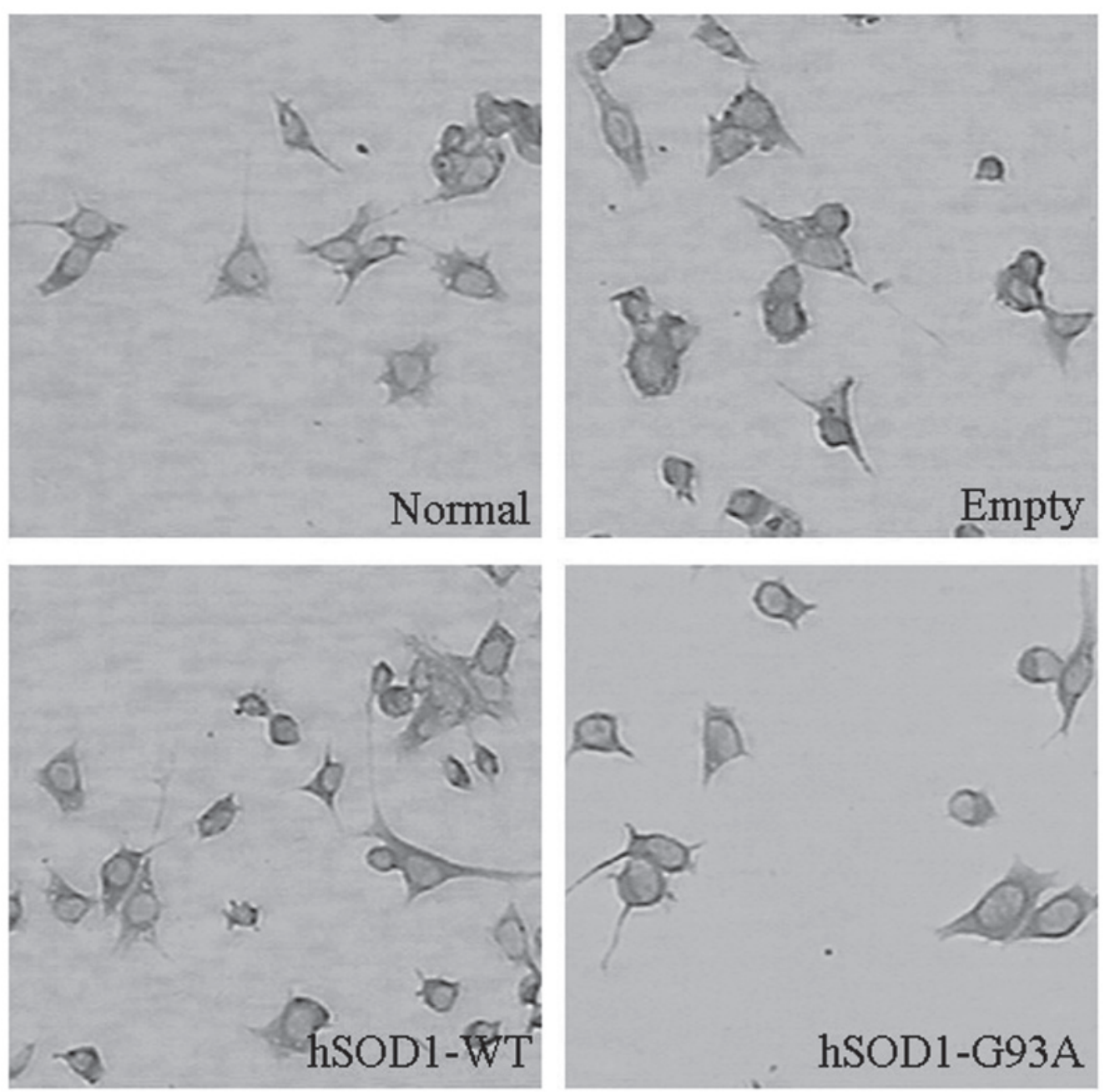

Figure 1. Immunocytochemical staining of cells. The cells were seeded on glass slides in a six-well culture plate and stained using SMI-32 (an anti-neurofilament monoclonal antibody). The four images are representative of immunostaining of cells with SMI-32 (magnification, x100). Normal, NSC-34 cell lines; Empty, NSC-34 cell lines transfected with the empty pcDNA3.1(-) plasmid; hSOD1-WT, NSC-34 cell lines transfected with the pcDNA3.1(-) plasmid carrying hSOD1-WT; hSOD1-G93A, NSC-34 cell lines transfected with the pcDNA3.1(-) plasmid carrying mutant hSOD1-G93A. hSOD1, human superoxide dismutase 1; WT, wild type.

which is a single electron reductase. Therefore, the process had a protective function against oxidative stress caused by metabolism. The current study suggested that destroying the Nrf2 molecules and its downstream signaling pathway was able to aggravate the deterioration of oxidative damage, inflammation and mitochondrial dysfunction in cells (22). Furthermore, several previous studies $(17,23)$ indicated that activated $\mathrm{Nrf} 2$ was beneficial to organisms in the context of hSOD1-G93A toxicity, and selective Nrf2 overexpression in neurons or type II skeletal muscle fibers was able to delay disease onset in hSOD1-G93A mice. Various previous studies revealed that phase II enzyme inducers were able to upregulate the expression of HO-1 and protect neurons from the damage caused by oxidative stress through Nrf2/ARE pathways $(24,25)$. The above evidence suggested that the Nrf2/ARE signaling pathway is able to protect against oxidative stress damage and subsequently protect the neurons in the ALS cell model. Therefore, in the present study, the cause of increased oxidative stress levels in the mutant hSOD1 of NSC-34 cells may be associated with the result that the human SOD1-G93A gene affected the Nrf2/ARE signaling pathway and reduced the expression of HO-1 and NQO1 in NSC-34 cells. In addition, these findings suggest that

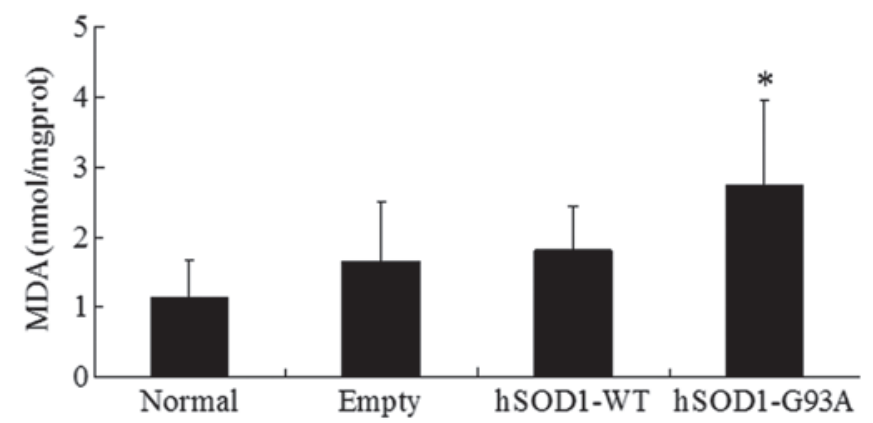

Figure 2. Quantitative assessment of the levels of MDA in four cell lines (mean \pm standard deviation; $\mathrm{n}=4$ ). ${ }^{*} \mathrm{P}<0.05$, statistically different from the normal, empty, hSOD1-WT cell lines. MDA, malondialdehyde; hSOD1, human superoxide dismutase 1 ; WT, wild type.

activating the Nrf2/ARE signaling pathway and increasing phase II enzymes are potential therapeutic strategies for the treatment of ALS.

In conclusion, the present study demonstrated that the human SOD1-G93A gene damaged the Nrf2/ARE signaling pathway, 
A

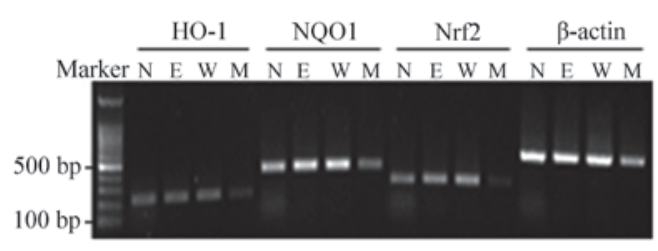

C

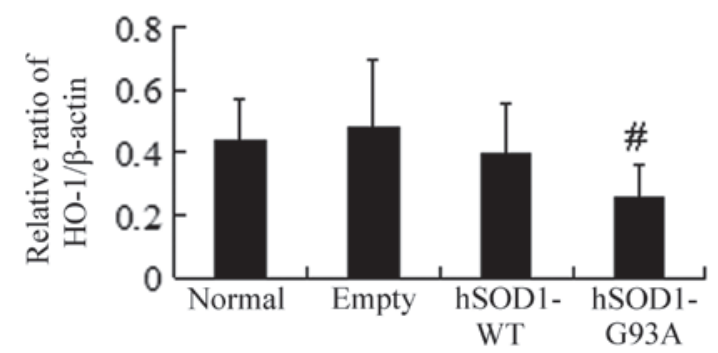

B

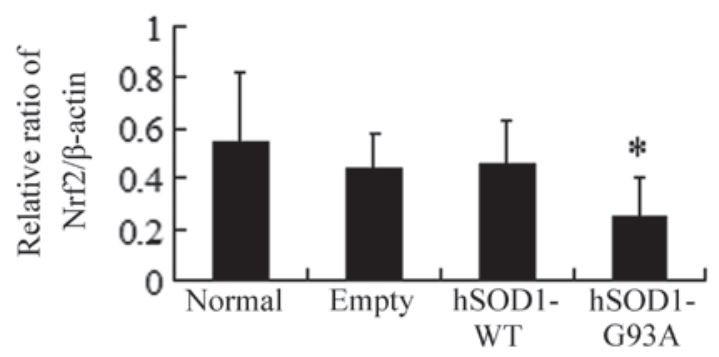

D

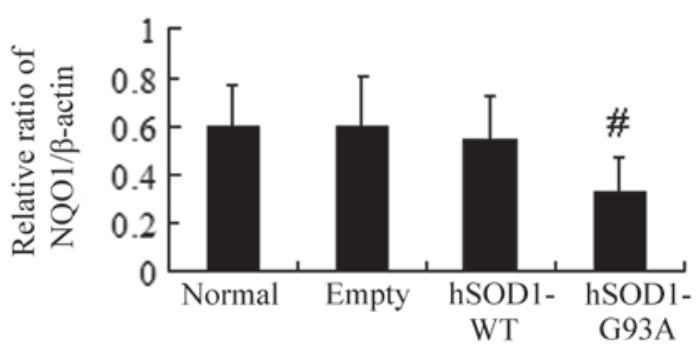

Figure 3. Analysis of the mRNA expression of Nrf2 and antioxidant response element-driven genes in four cell lines. (A) Total RNA was extracted from the four cell lines and HO-1, NQO1 and Nrf2 mRNA levels were determined by PCR and corrected by $\beta$-actin mRNA levels (N, normal: E, empty; W, hSOD1-WT; M, hSOD1-G93A). (B) Densities of Nrf2 bands were measured and its ratio to $\beta$-actin was calculated (mean \pm standard deviation; $n=6$ ). (C) Densities of HO-1 bands were measured and its ratio to $\beta$-actin was calculated (mean \pm standard deviation; $n=8$ ). (D) Densities of NQO1 bands were measured and its ratio to $\beta$-actin was calculated (mean \pm standard deviation; $\mathrm{n}=6$ ). ${ }^{*} \mathrm{P}<0.05$ and ${ }^{~} \mathrm{P}<0.01$, statistically different from the normal, empty, hSOD1-WT cell lines. Nrf2, nuclear factor erythroid 2-related factor 2; NQO1, NAD(P)H: quinone oxidoreductase 1; HO-1, heme oxygenase-1; WT, wild type; hSOD1, human superoxide dismutase 1.

A

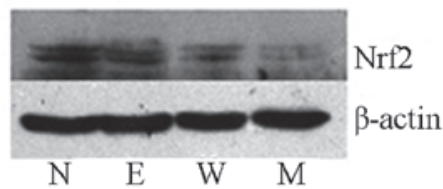

C

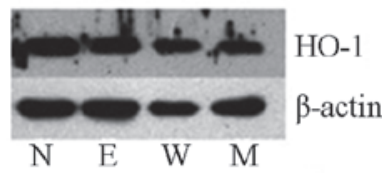

$\mathbf{E}$

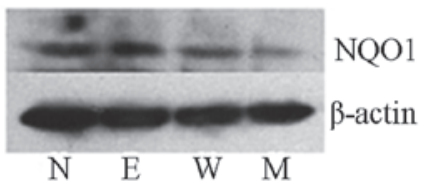

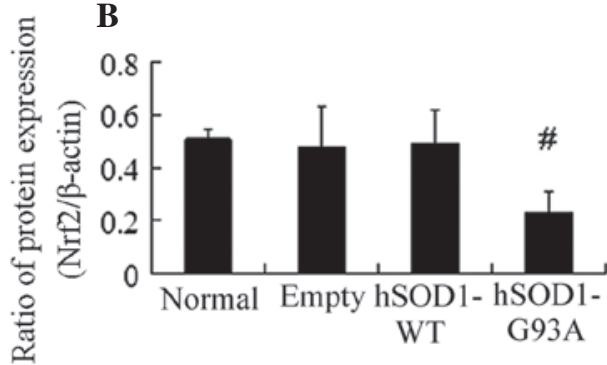

D
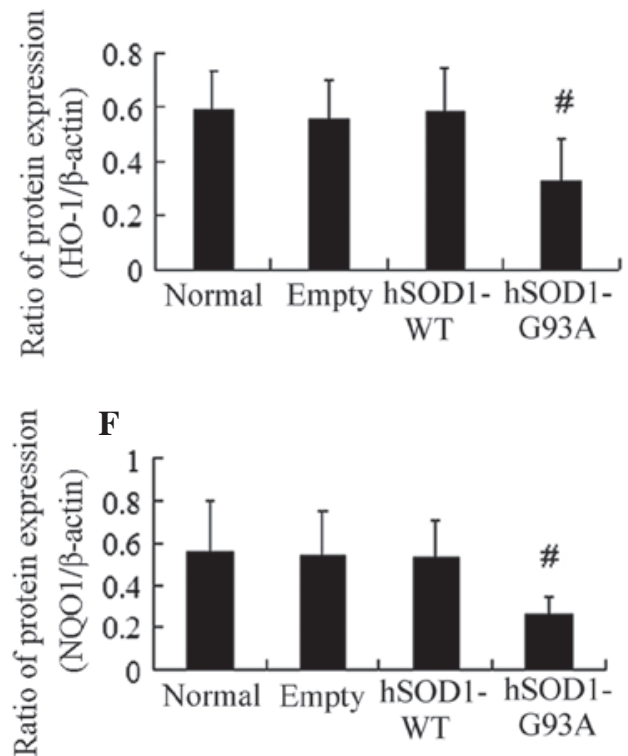

Figure 4. Western blot analysis to measure the protein expression levels of HO-1, NQO1 and Nrf2 in four cell lines. (A and B) Densities of Nrf2 bands were measured and its ratio to $\beta$-actin was calculated (mean \pm standard deviation; $\mathrm{n}=4$; N, normal; E, empty; W, hSOD1-WT; M, hSOD1-G93A). (C and D) Densities of HO-1 bands were measured and its ratio to $\beta$-actin was calculated (mean \pm standard deviation; $\mathrm{n}=6$ ). (E and F) Densities of NQO1 bands were measured and its ratio to $\beta$-actin was calculated (mean \pm standard deviation; $\mathrm{n}=6$ ). ${ }^{*} \mathrm{P}<0.01$, statistically different from the normal, empty, hSOD1-WT cell lines. Nrf2, nuclear factor erythroid 2-related factor 2; NQO1, NAD(P)H: quinone oxidoreductase 1; HO-1, heme oxygenase-1; WT, wild type; hSOD1, human superoxide dismutase 1. 
reduced the ability of cells to protect against oxidative damage and increased the vulnerability of motor neurons exposed to oxidative stress.

\section{Acknowledgements}

This study was supported in part by a grant from the Natural Science Foundation of Shandong, China (grant no. ZR2010HM041) and a grant from the Post-doctoral Innovation Foundation of Shandong, China (grant no. 201102004).

\section{References}

1. Avossa D, Grandolfo M, Mazzarol F, et al: Early signs of motoneuron vulnerability in a disease model system: characterization of transverse slice cultures of spinal cord isolated from embryonic ALS mice. Neuroscience 138: 1179-1194, 2006.

2. Mitchell JD, Callagher P, Gardham J, et al: Timelines in the diagnostic evaluation of people with suspected amyotrophic lateral sclerosis (ALS)/motor neuron disease (MND) - a 20-year review: can we do better? Amyotroph Lateral Scler 11: 537-541, 2010 .

3. Kiernan MC, Vucic S, Cheah BC, et al: Amyotrophic lateral sclerosis. Lancet 377: 942-955, 2011.

4. Zinman L and Cudkowicz M. Emerging targets and treatments in amyotrophic lateral sclerosis. Lancet Neurology 10: 481-490, 2011.

5. Barber SC and Shaw PJ: Oxidative stress in ALS: key role in motor neuron injury and therapeutic target. Free Radical Biol Med 48: 629-641, 2010.

6. Petri S, Körner S and Kiaei M: Nrf2/ARE signaling pathway: key mediator in oxidative stress and potential therapeutic target in ALS. Neurol Res Int 2012: 878030, 2012.

7. Valdmanis PN, Daoud H, Dion PA and Rouleau GA: Recent advances in the genetics of amyotrophic lateral sclerosis. Curr Neurol Neurosci Rep 9: 198-205, 2009.

8. Howland DS, Liu J, She Y, et al: Focal loss of the glutamate transporter EAAT2 in a transgenic rat model of SOD1 mutant-mediated amyotrophic lateral sclerosis (ALS). Proc Natl Acad Sci USA 99: 1604-1609, 2002.

9. Gurney ME, Pu H, Chiu AY, et al: Motor neuron degeneration in mice that express a human $\mathrm{Cu}, \mathrm{Zn}$ superoxide dismutase mutation. Science 264: 1772-1775, 1994.

10. Yu X and Kensler T: Nrf2 as a target for cancer chemoprevention. Mutat Res 591: 93-102, 2005.
11. Taguchi K, Motohashi H and Yamamoto M: Molecular mechanisms of the Keap1-Nrf2 pathway in stress response and cancer evolution. Genes Cells 16: 123-140, 2011.

12. Vargas MR and Johnson JA: The Nrf2-ARE cytoprotective pathway in astrocytes. Expert Rev Mol Med 11: e17, 2009.

13. Hybertson BM, Gao B, Bose SK and McCord JM: Oxidative stress in health and disease: the therapeutic potential of Nrf2 activation. Mol Aspects Med 32: 234-246, 2011.

14. Mariani E, Polidori MC, Cherubini A and Mecocci P: Oxidative stress in brain aging, neurodegenerative and vascular diseases: an overview. J Chromatogr B Analyt Technol Biomed Life Sci 827: 65-75, 2005.

15. Shibata N, Nagai R, Uchida K, et al: Morphological evidence for lipid peroxidation and protein glycoxidation in spinal cords from sporadic amyotrophic lateral sclerosis patients. Brain Res 917: 97-104, 2001.

16. Sarlette A, Krampfl K, Grothe C, et al: Nuclearerythroid2-related factor 2-antioxidative response element signaling pathway in motor cortex and spinal cord in amyotrophic lateral sclerosis. J Neuropathol Exp Neurol 67: 1055-1062, 2008.

17. Pehar M, Vargas MR, Robinson KM, et al: Mitochondrial superoxide production and nuclear factor erythroid 2-related factor 2 activation in p75 neurotrophin receptor-induced motor neuron apoptosis. J Neurosci 27: 7777-7785, 2007.

18. Neymotin A, Calingasan NY, Wille E, et al: Neuroprotective effect of Nrf2/ARE activators, CDDO ethylamide and CDDO trifluoroethylamide, in a mouse model of amyotrophic lateral sclerosis. Free Radic Biol Med 51: 88-96, 2011.

19. Malhotra D, Portales-Casamar E, Singh A, et al: Global mapping of binding sites for Nrf2 identifies novel targets in cell survival response through ChIP-Seq profiling and network analysis. Nucleic Acids Res 38: 5718-5734, 2010.

20. Poss KD and Tonegawa S: Reduced stress defense in heme oxygenase 1-deficient cells. Proc Natl Acad Sci USA 94: 10925-10930, 1997.

21. Siegel D, Gustafson DL, Dehn DL, et al: NAD(P)H:quinone oxidoreductase 1: role as a superoxide scavenger. Mol Pharmacol 65: 1238-1247, 2004.

22. Slocum SL and Kensler TW: Nrf2: control of sensitivity to carcinogens. Arch Toxicol 85: 273-284, 2011.

23. Vargas MR, Burton NC, Kutzke J, et al: Absence of Nrf2 or its selective overexpression in neurons and muscle does not affect survival in ALS-linked mutant hSOD1 mouse models. PLoS One 8: e56625, 2013.

24. Sun MM, Bu H, Li B, et al: Neuroprotective potential of phase II enzyme inducer diallyl trisulfide. Neurol Res 31: 23-27, 2009.

25. Stack C, Ho D, Wille E, et al: Triterpenoids CDDO-ethyl amide and CDDO-trifluoroethyl amide improve the behavioral phenotype and brain pathology in a transgenic mouse model of Huntington's disease. Free Radic Biol Med 49: 147-158, 2010. 